\title{
DETERMINAÇÃO DA CONDUTIVIDADE HIDRÁULICA DO LATOSOLO ROXO DISTRÓFICO POR ATENUAÇÃO DE RAIOS GAMA E TENSIOMETRIA
}

\author{
JÚLIO CÉSAR MARTINS DE OLIVEIRA ${ }^{1}$ \\ KLAUS REICHARDT ${ }^{2}$ \\ CARLOS ROBERTO APPOLONI ${ }^{3}$ \\ ANTÔNIO DOMINGOS BÁRTHOLO DE SOUZA \\ ANTÔNIO CARLOS SARAIVA DA COSTA ${ }^{5}$
}

OLIVEIRA, J.C.M.; REiCHARDT, K.; APPOLONI, CR.; SOUZA, A.D.B.; COSTA A.C.S. Determinação da condutividade hidráulica do latossolo roxo distrófico por atenuação de raios gama e tensiometria. Semina: Ci. Exatas/Tecnológicas, Londrina, v. 16, n. 4, p. 550-553, dez. 1995.

RESUMO: O presente trabalho apresenta o estudo da condutividade hidráulica de um latossolo roxo distrófico em experimentos de campo e laboratório. O experimento em laboratório simulou as condições de campo utilizando perfis de umidade em função do tempo em colunas de solo. Os dados foram obtidos pela atenuação de raios gama de uma fonte de ${ }^{241} \mathrm{Am}$, detectados através de equipamento padrão de espectrometria gama. No experimento de campo, foram utilizados tensiômetros nas profundidades de 10 e $25 \mathrm{~cm}$, para obtenção dos perfis de umidade em função do tempo. Os métodos de determinação da condutividade hidráulica foram os baseados na drenagem interna do solo. Os resultados mostraram maiores valores da condutividade hidráulica medida no campo, comparados com os valores de laboratório. Os métodos de determinação da condutividade hidráulica apresentaram valores distintos, tanto para os experimentos de campo como para os de laboratório.

PALAVRAS-CHAVE: condutividade hidráulica; atenuação gama.

\section{1 -INTRODUÇÃO}

A água é a substância de maior atividade cíclica da natureza. Atinge a superfície do soio querseja através de chuvas, quer seja por mecanismos não naturais como a irrigação Ao penetrar peia superfície do solo é por ele armazenada em seus poros ficando em parte disponível às plantas. Se a capacidade de armazenamento for ultrapassada, o excesso de água infiltrada se redistribui para horizontes mais profundos, podendo contribuir para a recarga de aqüíferos subterrâneos.

Para o pleno desenvolvimento das plantas e consequentemente altos níveis de produtividade, um dos principais fatores envolvidos é a disponibilidade de água. O estudo e a compreensão do papel que o armazenamento e a redistribuicão desempenham nesta disponibilidade é, portanto, de grande e real interesse. Assim são de suma importância ensaios de laboratório e de campo envolvendo processos como a redistribuição de água no solo, bem como a obtenção de algumas propriedades que controlam estes processos.

O presente trabalho teve como objetivo comparar os métodos e metodologias de determinação da condutividade hidráulica do soio em experimentos de campo e laboratório.

\section{2 - MATERIAL E MÉTODOS}

\section{1 - Condições de campo}

Para a determinação da condutividade hidráulica em condições de campo foram instalados tensiômetros em uma parcela de latossoio roxo distrófico (L.R.d.), de textura argilosa, no campo experimental do Instituto Agronômico do Paraná, Londrina, nas profundidades 10 e $25 \mathrm{~cm}$. A superfície da parcela foi inundada para que a infiltração em todo perfil entrasse em equilíbrio dinâmico, o que foi observado através da estabilidade das leituras nos tensiômetros.

Após a infiltração da lâmina, cobriu-se a parcela com plástico para evitar a evaporação. Leituras periódicas dos tensiômetros foram feitas durante 25 dias.

Para elaboração da curva característica de retenção da água foram coletadas três amostras, em cada profundidade, com estrutura indeformada ao lado da parcela. Com as leituras feitas nos tensiômetros e com as curvas características da água do solo, foram obtidos os respectivos valores de umidade do soío. Para as mesmas amostras utilizadas na elaboração da curva característica, determinou-se também a densidade, a porosidade total, macro e microporosidade (Kiehl, 1979).

1. Seção de Física de Solos / CENA/USP, Caixa Postal 96, Piracicaba, SP, CEP 13400-970, Fone: (0194) 335122 r. 217.

2. Departamento de Física/ESALQ/USP, Caixa Postal 96, Piracicaba, SP, CEP 13400-970.

3. Departamento de Física / Universidade Estadual de Londrina, Caixa Postal 6001, Londrina, PR, CEP 86051-970.

4. Centro Federal de Educação Tecnológica, Av. Alberto Carazzaí, 1640. Cornélio Procópio, PR, CEP 86300-000.

5. Departamento de Agronomia / Universidade Estadual de Maringá, Caixa Postal 331, Maringá, PR, CEP 87020-900. 
A partir de dados de umidade do solo em função dos tempos de redistribuição aplicou-se os métodos de Hillel et al. (1972) e o de Libardi et al. (1980) para o cálculo da condutividade hidráulica.

\section{2 - Condições de laboratório}

Utilizou-se amostras das camadas de 0 a 10 e 10 a $25 \mathrm{~cm}$ do mesmo solo (L.R.d.), as quais foram secas ao ar e passadas em peneira de $2 \mathrm{~mm}$. A partir dos dados de densidade do solo no campo, determinou-se a quantidade de solo necessária para preencher os cilindros de $50 \mathrm{~cm}^{3}$, utilizados na elaboração da curva característica da água do solo com estrutura deformada, simulando condições de campo. As mesmas condições foram aplicadas nas colunas de acrílico: $90 \mathrm{~cm}$ de comprimento e $5 \mathrm{~cm}$ de diâmetro, empregadas nas medidas de infiltração e redistribuição em laboratório.

Para determinação da densidade e umidade do solo em laboratório utilizou-se o sistema clássico de atenuação de radiação gama, com movimentação da fonte e do detector, mantendo-se a coluna de solo fixa (Figura 1).

As colunas foram selecionadas para a infiltração de água utilizando-se como critério de homogeneidade

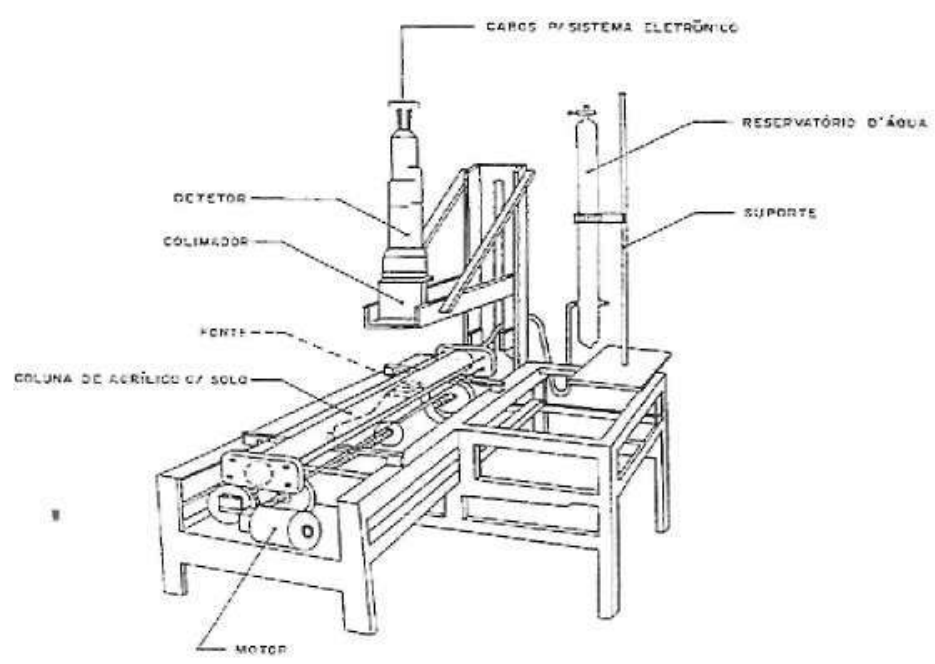

Figura 1 - Sistema de infiltração utilizado para as medidas em laboratório. a distribuição de densidade do solo, medida a cada 2 $\mathrm{cm}$ na coluna de solo. O processo de infiltração horizontal foi cessado quando a frente de molhamento atingiu aproximadamente $30 \mathrm{~cm}$ de profundidade na coluna de solo. Iniciou-se então o processo de redistribuição, com a coluna na posição vertical para que a água pudesse drenar. Foram feitas medidas de atenuação nas posições 10 e $25 \mathrm{~cm}$ das colunas de solo para os vários tempos de redistribuição durante 25 dias. Posteriormente obteve-se os perfis de umidade em função dos tempos de redistribuição. Para estes dados também aplicou-se os métodos de Hiilel et al. (1972) e Libardi et al. (1980).

\section{3 - RESULTADOS E DISCUSSÃO}

Os valores médios de densidade do solo no campo e nas colunas de laboratório, bem como seus respectivos desvios padrões são mostrados na Tabela 1. Podemos observar que o acondícionamento de solo nas colunas foi satisfatório comparando os valores de densidade do solo com os valores de campo. A Figura 2 mostra a distribuição da densidade do solo nas colunas (até 30 $\mathrm{cm}$ de comprimento) para as amostras coletadas nas duas profundidades de interesse $(0-10$ e $10-25 \mathrm{~cm})$.

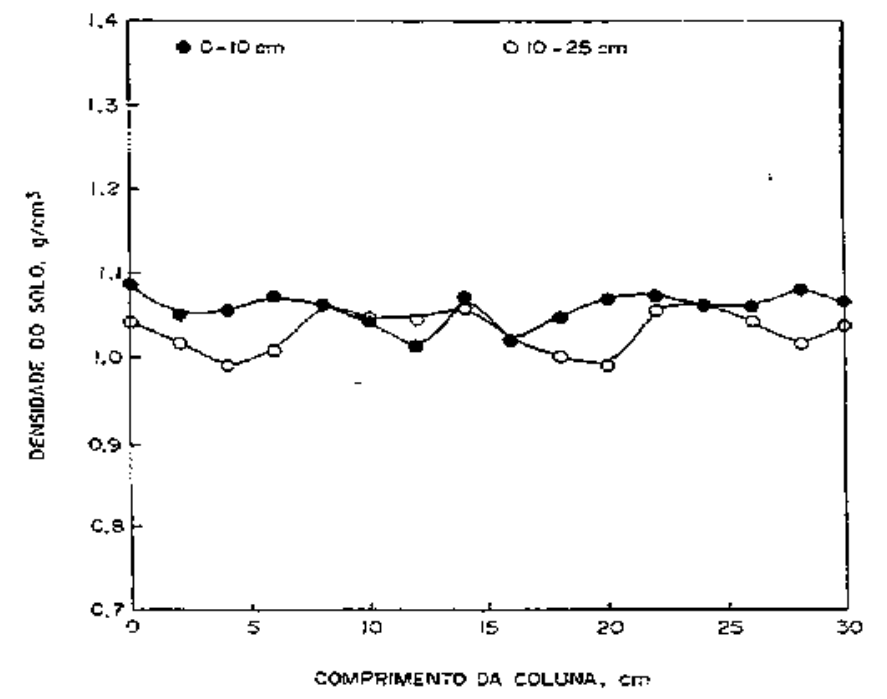

Figura 2 - Distribuição da densidade do solo em funçăo do camprimento das colunas para amostras calibradas nas camadas de $0-10$ e $10-25 \mathrm{~cm}$.

Tabela 1 - Valores médios da umidade do solo seco ao ar $\left(\theta_{\mathrm{O}}\right)$, densidade do solo $\left(\rho_{\mathrm{S}}\right)$, seu desvio padrão $(\sigma)$ no campo e em colunas no laboratório, para o latossolo roxo distrófico.

\begin{tabular}{llllll}
\hline Profundidade & \multicolumn{2}{c}{ Campo } & \multicolumn{2}{c}{ Laboratório } \\
& $\theta_{\mathbf{O}}$ & $\rho_{\mathbf{S}}$ & $\sigma$ & $\rho_{\mathbf{s}}$ & $\sigma$ \\
$\mathbf{c m}$ & $\mathbf{c m}^{3 /} / \mathbf{c m}^{3}$ & $\mathbf{g} / \mathbf{c m}^{3}$ & & 1,04 & 0,04 \\
10 & 0,068 & 1,10 & 0,07 & 0,98 & 0,05 \\
\hline
\end{tabular}


Os valores da porosidade total, macro e microporosidade, para campo e laboratório, são mostrados na Tabela 2. Podemos observar que a porosidade total em condições de campo e laboratório praticamente não se alterou, mas a relação entre macro e microporosidade mostrou-se diferente. Em condições de laboratório a porcentagem de macroporos diminuiu e consequentemente a microporosidade aumentou comparando com os valores de campo. Assume-se como justificativa disto o fato das amostras terem sido peneiradas, destruindo a estrutura natural modificando a agregação do solo diminuindo a sua macroporosidade.

Tabela 2 - Valores da densidade do solo, densidade de particulas, porosidade total, macro e microporosidade para o latossojo roxo distrófico em condições de campo e laboratório.

\begin{tabular}{lllllll}
\hline Tratamento & $\begin{array}{c}\text { Prof. } \\
\mathbf{c m}\end{array}$ & $\begin{array}{l}\text { Densidade } \\
\mathbf{d o} \mathbf{s o l o}^{\mathbf{3}} \\
\mathbf{9 / \mathbf { c m } ^ { 3 }}\end{array}$ & $\begin{array}{l}\text { Densidade de } \\
\text { particulas } \\
\mathbf{g} / \mathbf{c m}^{\mathbf{3}}\end{array}$ & $\begin{array}{l}\text { Porosidade } \\
\text { total } \\
\%\end{array}$ & $\begin{array}{l}\text { Micro } \\
\text { porosidade } \\
\%\end{array}$ & $\begin{array}{l}\text { Macro } \\
\text { porosidade } \\
\%\end{array}$ \\
\hline Campo & 10 & 1,10 & 3,03 & 64 & 48 & 52 \\
& 25 & 1,14 & 3,07 & 63 & 60 & 50 \\
Laboratório & 10 & 1,04 & 3,03 & 66 & 71 & 29 \\
& 25 & 0,98 & 3,07 & 68 & 62 & 38 \\
\hline
\end{tabular}

A Figura 3 mostra os dados da condutividade hidráulica em função da umidade do solo, obtidos nos experimentos de campo e laboratório utilizando o método de Hillel et al. (1972). Podemos observar que os valores

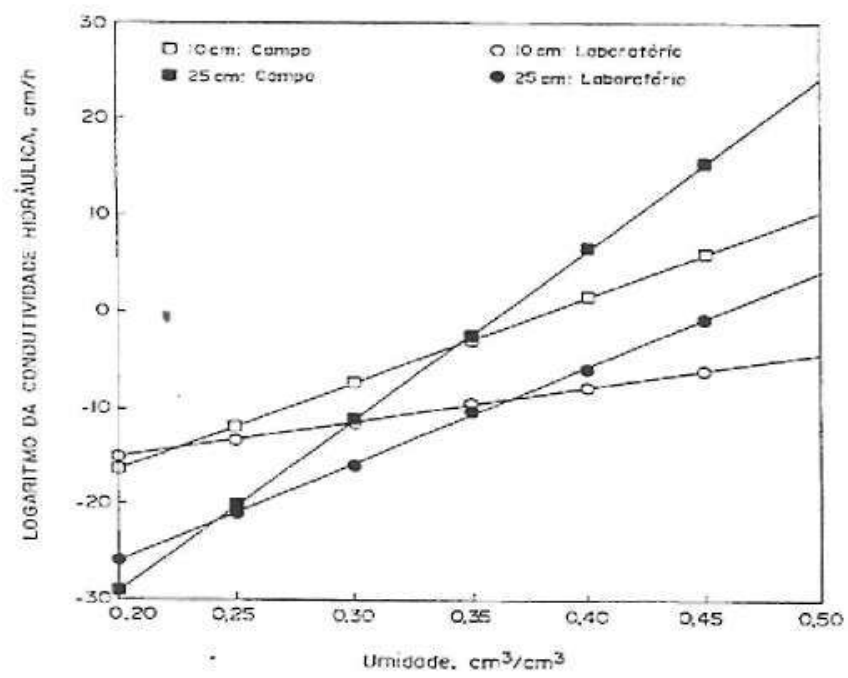

Figura 3 - Relação entre a condutividade hidráulica e a umidade obtidas no campo e no laboratório, utilizando o método de Hiliel et al. (1972).

A Figura 5 mostra a comparação dos métodos de Hillel et al. (1972) e Libardi et al. (1980). Podemos observar que a condutividade é maior quando calculada pelo método de Hillel et al. (1972) para as duas de condutividade hidráulica em condições de campo foram maiores para o mesmo valor de umidade nas duas profundidades estudadas. Isso também foi verificado para o método de Libardi et al. (1980), como mostra a Figura 4.

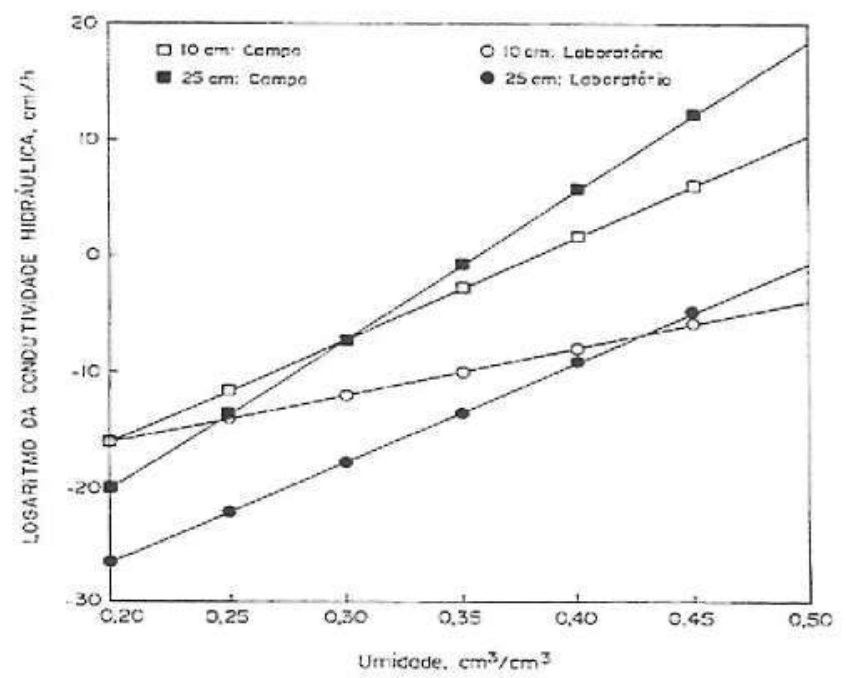

Figura 4 - Relaçäo entre condutividade hidráulica e a umidade obtidas no campo e laboratório, utilizando o método de Libardi et al. (1980).

profundidades, para um mesmo valor de umidade; isso foi observado também na comparação dos métodos nos experimentos em condições de campo como mostra a Figura 6. 


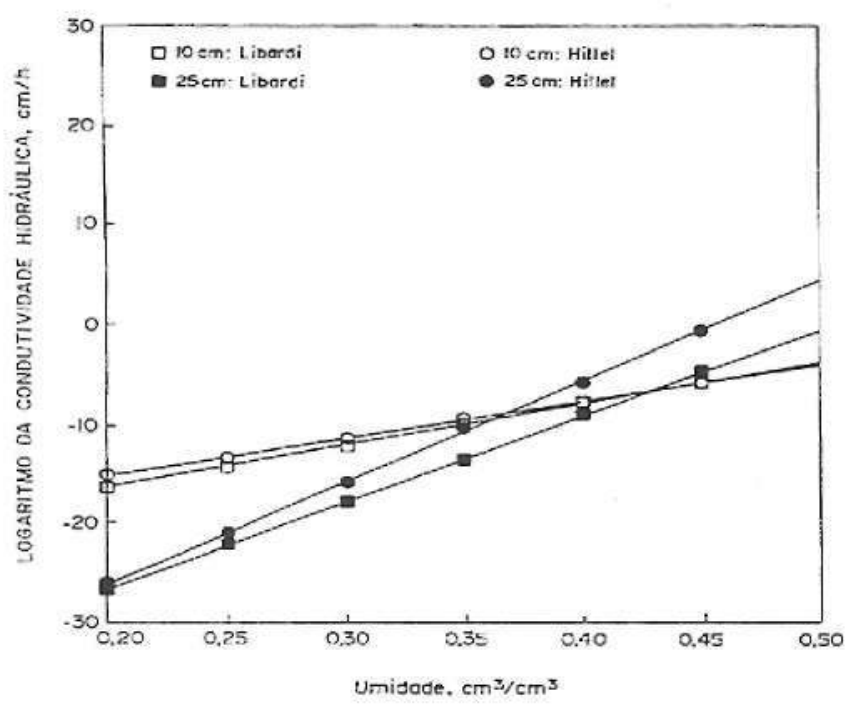

Figura 5 - Comparação entre o método de Libardi et al. (1980) e de Hillel et al. (1972) na determinação da condutividade hidráulica em função da umidade nos experimentos de laboratório.

O método de Hillel et al. (1972) mostrou-se mais simples comparado com o de Libardi et al. (1980) para a determinação da condutividade hidráulica quando considera-se o gradiente do potencial como sendo unitário, Apesar dessa facilidade o método de Hillel et al. (1972) difere do de Libardi et al. (1980) quando analisamos a determinação da condutividade hidráulica saturada $\left(\mathrm{K}_{0}\right)$. No primeiro método $\mathrm{K}_{0}$ é obtida por regressões dos resultados finais na forma In $\mathrm{K}(\mathrm{q})$ vs q e no segundo a constante $\mathrm{K}_{0}$ é obtida diretamente das regressões ( $\mathrm{q}-\mathrm{q}_{0}$ ) vs In t. Esta diferença pode ter influído nos diferentes valores obtidos para a condutividade

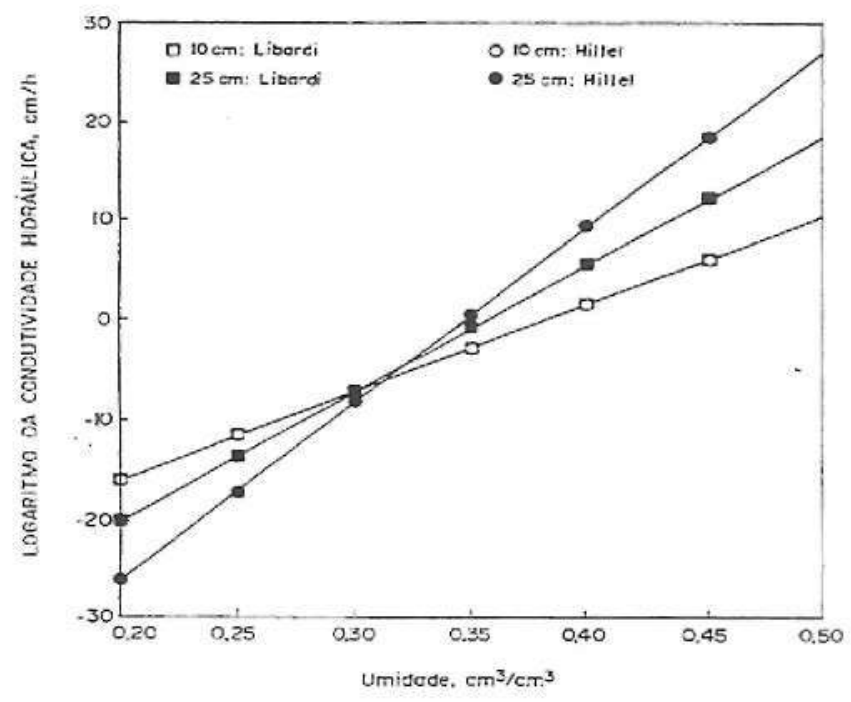

Figura 6 - Comparação entre o método de Libardi et al. (1980) e de Hillel et al. (1972) na determinaçâo da condutividade hidráulica em função da umidade nos experimentos de campo.

hidráulica quando se compara os métodos de Hillel et al. (1972) e Libardi et al. (1980), tanto nos experimentos de campo como nos de laboratório.

\section{4-CONCLUSÃO}

A condutividade hidráulica do solo, determinada por tensiometria no campo foi maior do que a obtida por atenuação de raios gama, em amostras deformadas (laboratório), o que compromete a extrapolação dos resultados de laboratório para a situação de campo.

OLIVEIRA, J.C.M.; REICHARDT, K.; APPOLONI, CR.; SOUZA, A.D.B.; COSTA, A.C.S. Hydraulic conductivity determination of a dark red latosol by gamma attenuation and tensiometry. Semina: CL Exatas/Tecnológicas, Londrina, v. 16, n. 4, p. 550-553, Dec. 1995.

ABSTRACT: Results for the hydraulic conductivity of a dark red latosol (Oxisol) under laboratory and field conditions are presented. The laboratory experiments simulated field conditions through the measurement of the soil water content profiles as a function of time in soil columns. The data were obtained by the ${ }^{241}$ Arn gamma-ray transmission method, using standard gamma ray spectrometry equipament. Tensiometers at the depths of 10 and $25 \mathrm{~cm}$ were used to obtain the soil water content profiles as a function of time in the field experiments. The hydraulic conductivity functions were determined through internal soil drainage. The results showed higher values of the hydraulic conductivity measured in the field, compared with the laboratory values. The hydraulic conductivity determination methods presented distinct values for the field experiments as well as for the laboratory ones.

KEY-WORDS: hydraulic conductivity; gamma attenuation,

\section{REFERÊNCIAS BIBLIOGRÁFICAS}

HILLEL, D.; KRENTOS, V.D, \& STYLIANOU, Y. Procedure and test for on internal drainage method for measuring soil hydratulic characteristics in situ. Soil Sci. Soc. Am. Proc., Madison, 114: 395-400, 1972.

KIEHL, E.J. Manual de edafologia: relações solo-planta.
Sầ Paulo, Ceres, 1972, p. 262

LIBARDI, P.L.; REICHARDT, K.; NIELSEN, D.R. \& BIGGAR, JW. Simplified methods for estimating the unsaturated nydraulic conductivity. Soil Sci. Soc. Am. J., Madison, 44: 3-7, 1980. 